\title{
Comunicación y educación. Análisis de la demanda de egresados de la licenciatura en comunicación social en organizaciones
}

\author{
Recibido: 07 de septiembre de 2016 \\ Aceptado: 10 de abril de 2017 \\ Publicado: 27 de noviembre de 2017
}

\author{
Alejandra María Gordillo \\ mgordillo@undec.edu.ar \\ Universidad Nacional de Chilecito (Argentina)
}

\begin{abstract}
Resumen: La reflexión “comunicación-educación” no se limita al análisis de las prácticas, las metodologías de enseñanza-aprendizaje, el rol de los sujetos en la díada o la cuestión ideológica que atraviesa las políticas educativas, sino que pone en debate la tríada "docenteconocimiento-estudiante" en cada momento y contexto histórico. El binomio al que se alude puede y debe ser enriquecido también a partir de la indagación científica, que dé cuenta (entre otros tantos aspectos) de la efectiva inserción de los profesionales (concebido como el fruto de ese proceso formativo de la "comunicación-educación") en el entorno organizacional inmediato de actuación de la institución educativa de nivel superior. Con ese objetivo en vista, se llevó a cabo una investigación exploratoria (como un primer y necesario paso) para conocer más acerca de la demanda de comunicadores sociales en organizaciones (públicas, privadas o mixtas) en la ciudad de Chilecito, provincia de La Rioja (Argentina), espacio en el que se inserta la Universidad Nacional de Chilecito, en el período 2013-2014.
\end{abstract}

Las organizaciones son espacios de intervención profesional de los comunicadores sociales con orientación en comunicación institucional y es en ese ámbito tan amplio y complejo de realidades (socioculturales, administrativas, económicas, financieras, políticas e ideológicas diversas) donde pueden desplegar la versatilidad de las competencias y habilidades adquiridas en su formación de grado. Por otra parte, investigar sobre las características de la demanda de estos profesionales constituye un recurso valioso al momento de reconocer la viabilidad y la pertinencia de la oferta educativa. Estos aspectos han sido las principales motivaciones que han guiado la elaboración de la presente investigación.

Palabras clave: Comunicación, educación, instituciones educativas de nivel superior, organizaciones, demanda organizacional. 
Abstract: Reflection "Communication-Education" is not limited to the analysis of practices, teaching-learning methodologies, the role of subjects in the dyad or the ideological issue that crosses the educational policies, but discuss the triad "teacher-knowledge-student" every time in a historical context. The binomial referred should also be enriched by scientific inquiry, which accounts for (among many other aspects) effective job placement of professionals (conceived as the fruit of this educational process of "Communication-Education") in the immediate organizational environment of the higher education institutions. With this objective in view, an exploratory research was carried out (as a first and necessary step) to know more about the demand of social communicators in organizations (public, private or mixed) in the city of Chilecito, province of La Rioja (Argentina), an area which National University of Chilecito is inserted in period 2013-2014.

Organizations are areas of professional intervention for Social Communicators with Institutional orientation: it is a wide and complex area of socio-cultural, administrative, economic, financial, political and ideological realities where they can deploy versatility of the competences and skills acquired during their degree training. On the other hand, investigating characteristics of the demand of these professionals constitutes a valuable resource at the moment of recognizing the feasibility and suitability of educational offer. These aspects have been the main motivations that have guided the development of the present paper.

Key words: Communication, Education, Higher Education Institutions, Organizations, Organizational Demands.

\section{Introducción}

Este trabajo da cuenta de los resultados obtenidos en el marco del proyecto de investigación "Análisis de la demanda organizacional de licenciados en comunicación social en la ciudad de Chilecito". El interés central consistió en analizar las características de la demanda de comunicadores sociales en las organizaciones públicas y privadas de cierta complejidad administrativa radicadas en la ciudad de Chilecito, departamento del mismo nombre, en la provincia de La Rioja. La elección del lugar se funda en el hecho que en esta misma ciudad está ubicada la Universidad Nacional de Chilecito, cuya oferta académica incluye la licenciatura en comunicación social, con dos orientaciones: a) periodismo y b) publicidad y comunicación institucional.

Se buscó conocer si la dirección de las organizaciones locales reconocía la necesidad de profesionalizar las actividades de comunicación, entendida esta como un factor insustituible de la dinámica organizacional. Asimismo, recabar datos sobre el nivel de conocimientos que los actores organizacionales poseían sobre los alcances de la formación del comunicador institucional y su capacidad de operar en entornos que (aun siendo tan diversos entre sí) requieren de la comunicación para poder existir. Por último se indagó sobre la presencia efectiva de comunicadores sociales en esos espacios y sobre las actividades que desarrollaban. 


\section{Metodología}

Se trata de un trabajo de tipo exploratorio. Las unidades de análisis fueron las organizaciones de la ciudad de Chilecito, La Rioja, que por su tipología y complejidad administrativa se presumía debían requerir un comunicador social. Por ello, en una primera etapa, se seleccionaron las unidades de análisis, tipificándolas según su misión y su complejidad, es decir, las características administrativas y funcionales que puedan justificar la inserción labor de un profesional de la comunicación. Tomando en cuenta este criterio, se seleccionaron de manera aleatoria 33 organizaciones entre las que se encontraban las tipologías públicas, privadas y mixtas de todos los rubros de actividades: productivas, comerciales, de servicios, educativas, financieras, de turismo, comerciales, gremiales y otras.

En una segunda etapa, se aplicó una encuesta que constaba de preguntas en su mayoría cerradas y algunas abiertas. El cuestionario se centró en la indagación de las siguientes dimensiones: a) características generales de las organizaciones, entre las que se definieron las siguientes variables: jerarquía, tipo, actividad principal; b) la estructura organizacional y como desprendimiento de esta dimensión: la delegación de las actividades de comunicación, las profesiones en las que está delegada esta tarea, los criterios de selección y en quien recae esta decisión, la dependencia funcional y orgánica; c) acciones de comunicación interna y externa que desarrollan; y d) la demanda de licenciados en comunicación social. El método de análisis de los datos fue del tipo descriptivo e inferencial. En particular, tablas de frecuencia, gráficas y análisis de asociación chi-cuadrado, utilizando el software estadístico INFOSTAT.

\section{Lo comunicacional y lo organizacional}

La comunicación es básicamente una actividad humana, anclada en un sistema lingüístico de significantes y significados que aportan sentido y permiten la vinculación de los sujetos entre sí en un marco de comprensión y adhesión que aportan sentido a la trama sociocultural, facilitando la convivencia. Se asume como una práctica social cotidiana que permite la puesta en común de un mensaje expresado a diversos medios (oral, escrito, visual, audiovisual, gestual, tecnológico), utilizando un código (sistema de signos y símbolos compartido, comprensible para ambos integrantes del proceso comunicativo, es decir, emisor y receptor) que vincula a dos o más personas entre sí, generando una interacción, una interactuación, un vínculo comunicativo entre unos y otros.

Para Pichon Rivière, se trata de un proceso de interacción social por medio de símbolos y mensajes, que incluye todo proceso en el cual la conducta de un ser humano actúa como estímulo de la conducta de otro ser humano, pudiendo desenvolverse de manera verbal o no verbal, inter individual o intergrupalmente (ápud Schvarstein, 1998). De esta forma, es una actividad social en la que se producen, transmiten y receptan interpretaciones y significaciones de la realidad, enmarcadas en un contexto específico y compartido de convenciones para denominar sus múltiples aristas. 
Los sujetos individuales y colectivos (actores organizacionales) se relacionan entre sí mediante nexos comunicativos, poniendo en juego procesos mentales, psicológicos, corporales y culturales. Cada vez que se interviene en un proceso comunicacional no sólo se están presentes las expresiones verbales, sino también las gestuales, posturales, corporales, icónicas a las que cada quien recurrirá y seleccionará buscando una determinada reacción en su interlocutor.

Pero, tratándose de un proceso en el que al menos dos intervienen, no es posible circunscribir el análisis sólo en la emisión, sino también considerar la recepción, porque allí también se producen actividades mentales, sensitivas y emocionales, que dan lugar al despliegue de herramientas interpretativas y mecanismos de respuesta frente al estímulo que motivan los mensajes.

Por esto, hablar de comunicación es asumir un rico y complejo proceso individual y colectivo de interactuación, que trasladado al ámbito organizacional, donde numerosos sujetos (actores organizacionales) comparten espacios y procesos vinculados en una trama estructural y funcional de roles y funciones, de mensajes ascendentes, descendentes y transversales, que otorgan a la dinámica comunicacional una mayor complejidad y una especificidad propias de la comunicación organizacional o comunicación institucional, como un desprendimiento del campo disciplinar de la comunicación social: "La comunicación es un fenómeno importante, esencial y sustantivo de las organizaciones. Sin comunicación las organizaciones no podrían existir" (Peiró y Bresó, 2012: 42).
"Las organizaciones son una creación humana, un producto social que nace como respuesta a la necesidad de satisfacer la demanda de bienes y servicios de la sociedad. Las organizaciones son entes creados por el hombre, son un artificio que permite adaptarlo a sus fines y también regula la conducta individual y social [...] La palabra organización aparece como un sistema social o como un conjunto de personas en relación de interdependencia y surge una estructura compuesta por elementos de lenta modificación, la estructura genera un proceso organizativo con elementos en constante cambio" (Álvarez, 2003: 123).

En ellas, los valores, la misión, visión, objetivos, funciones, distribución de roles y coordinación de actividades necesarias para alcanzar sus metas, requieren un tratamiento comunicacional y un estilo y tipo de comunicación propio de cada situación.

La sociedad actual puede ser concebida como una sociedad organizacional, por cuanto presenta una conformación multifacética, con una amplia y diversa integración de organizaciones que la integran. Dichas organizaciones son de diverso tipo y cumplen variadas funciones, e incluso muchas de ellas están íntimamente interrelacionadas, en mutua interdependencia. Coexisten e interactúan entre sí para satisfacer las crecientes demandas de una economía cada vez más diversificada.

"se encuentran dentro de esta categoría las empresas de todo tipo, las escuelas, los hospitales, los entes gubernamentales, las organizaciones no gubernamentales, los clubes, las asociaciones, el ejército, los sindicatos, las iglesias, las universidades, entre tantas otras. Están tan vinculadas entre 
sí y las personas a ellas, que a lo largo de la vida todas integran de una manera $u$ otra, y por un tiempo determinado, alguna organización. Se trata de un conjunto interrelacionado de actividades entre dos o más personas que interactúan para procurar el logro de un objetivo común, a través de una estructura de roles y funciones y una división del trabajo" (Krieger, 2001: 3).

El logro de objetivos comunes es el norte de todas las organizaciones, condición que le otorga sentido a la actividad integrada y conjunta de quienes la integran; razón necesaria y suficiente para que se coordinen actividades y se definan roles y funciones. Se requiere de un principio de orden y de coordinación que facilite la tarea de todos para alcanzar con éxito los propósitos por los cuales fue creada.

Schein (1988) concibe a la organización como una coordinación planificada de las actividades de un grupo de personas para procurar el logro de un objetivo o propósito explícito y común, lo cual se logra a partir de la división de trabajo y funciones, así como a través de una jerarquía de autoridad y responsabilidad. Para Mayntz (1980), se trata más bien de un sistema social encaminado hacia un objetivo, en acción recíproca con el medio, con tendencia a la autopreservación y a la integración.

En la sociedad es posible encontrar diversos tipos de organizaciones (figura 1), las que pueden ser clasificadas según los siguientes criterios:

Figura 1: Tipos de organizaciones.

\begin{tabular}{|c|c|c|}
\hline \multirow{3}{*}{$\begin{array}{l}\text { Según las } \\
\text { funciones que } \\
\text { desempeñan en la } \\
\text { sociedad }\end{array}$} & $\begin{array}{l}\text { Organización de } \\
\text { producción }\end{array}$ & Elaboran productos para consumo de la sociedad. \\
\hline & Organización política & $\begin{array}{l}\text { Se conforman para conducir los proyectos políticos y distribuir el } \\
\text { poder. }\end{array}$ \\
\hline & $\begin{array}{l}\text { Organización para } \\
\text { el mantenimiento de } \\
\text { patrones }\end{array}$ & $\begin{array}{l}\text { Son aquellas que buscan asegurar la continuidad de la sociedad a } \\
\text { través de actividades educativas, culturales, expresivas. }\end{array}$ \\
\hline \multirow{4}{*}{$\begin{array}{l}\text { Según las metas y } \\
\text { funciones } \\
\text { (Katz y Kahn) }\end{array}$} & $\begin{array}{l}\text { Organización } \\
\text { de producción o } \\
\text { económica }\end{array}$ & $\begin{array}{l}\text { Su función es crear capital, producir bienes o prestar servicios, como } \\
\text { empresas, bancos, mutuales, cooperativas, fábricas, etc. Pueden ser de } \\
\text { nivel primario, secundario o terciario. }\end{array}$ \\
\hline & $\begin{array}{l}\text { Organización de } \\
\text { mantenimiento }\end{array}$ & $\begin{array}{l}\text { Se dedican a la preparación de las personas para su inserción } \\
\text { normativa en la sociedad o en otras organizaciones. Se subdividen } \\
\text { en aquellas que se ocupan directamente del mantenimiento } \\
\text { (iglesias, escuelas) y en las que se encargan de restauración del } \\
\text { mantenimiento social (hospitales), de rehabilitación o reforma } \\
\text { (cárceles, centros de rehabilitación). }\end{array}$ \\
\hline & $\begin{array}{l}\text { Organizaciones } \\
\text { adaptantes }\end{array}$ & $\begin{array}{l}\text { Son aquellas que crean conocimiento, desarrollan nuevas teorías, brindan } \\
\text { soluciones a la sociedad a partir de la investigación y la vinculación, } \\
\text { como es el caso de las universidades. }\end{array}$ \\
\hline & $\begin{array}{l}\text { Organizaciones } \\
\text { administrativas o } \\
\text { politicas }\end{array}$ & $\begin{array}{l}\text { Son las encargadas de asignar, coordinar y controlar los recursos, } \\
\text { personas y subsistemas (Estados). }\end{array}$ \\
\hline
\end{tabular}




\begin{tabular}{|c|c|c|}
\hline \multirow{8}{*}{$\begin{array}{l}\text { Según los } \\
\text { objetivos que } \\
\text { persiguen } \\
\text { (Mayntz) }\end{array}$} & $\begin{array}{l}\text { Organizaciones } \\
\text { voluntarias }\end{array}$ & $\begin{array}{l}\text { Se conforman de común acuerdo entre los integrantes, persiguen } \\
\text { objetivos orientados a la actuación conjunta. Suelen tener una } \\
\text { organización democrática y las decisiones que adoptan son por } \\
\text { consenso o votación. En este grupo se nuclean los clubes, las } \\
\text { asociaciones, las cooperadoras. }\end{array}$ \\
\hline & $\begin{array}{l}\text { Organizaciones } \\
\text { estructuradas con el } \\
\text { objetivo de actuar } \\
\text { de una manera } \\
\text { determinada sobre un } \\
\text { grupo de personas: }\end{array}$ & $\begin{array}{l}\text { En esta categoría se ubican las escuelas, universidades, hospitales, } \\
\text { iglesias, cárceles, etc. La incorporación a estas organizaciones } \\
\text { puede ser voluntaria o forzosa, sus miembros pueden ser } \\
\text { permanentes o transitorios. La movilidad o cambio de roles suele } \\
\text { ser poco frecuente o nula (alumno-docente, carcelero-preso). }\end{array}$ \\
\hline & \multirow{4}{*}{$\begin{array}{l}\text { Organizaciones que } \\
\text { tienen por objetivo } \\
\text { el logro de cierto } \\
\text { resultado }\end{array}$} & $\begin{array}{l}\text { Con fines de lucro, como las empresas, cuyo fin constitutivo es la } \\
\text { obtención de una rentabilidad o beneficio económico. }\end{array}$ \\
\hline & & $\begin{array}{l}\text { En orden al bien común, como es el caso de la administración pública, } \\
\text { organizaciones benéficas (Cáritas), ONG. }\end{array}$ \\
\hline & & $\begin{array}{l}\text { En orden al bien de sus afiliados, como los sindicatos, las } \\
\text { asociaciones, las mutuales. Son organizaciones estructuradas } \\
\text { democráticamente, las decisiones se adoptan por consenso o } \\
\text { votación, la elección de sus dirigentes suele ser tomada en asamblea } \\
\text { general donde todos tienen derecho a elegir y ser elegidos. }\end{array}$ \\
\hline & & $\begin{array}{l}\text { En orden a la obtención de poder, tal el caso de los partidos } \\
\text { políticos o similares. También en éstas las decisiones suelen ser } \\
\text { democráticas, la afiliación voluntaria y sus integrantes ejercen el } \\
\text { derecho a elegir y ser elegidos. }\end{array}$ \\
\hline & \multirow{2}{*}{$\begin{array}{l}\text { Organizaciones } \\
\text { mixtas que combinan } \\
\text { características } \\
\text { de más de una } \\
\text { categoría: }\end{array}$} & $\begin{array}{l}\text { Las cooperativas, porque persiguen un fin lucrativo pero su } \\
\text { adhesión es voluntaria, poseen un gobierno democrático, las } \\
\text { decisiones de adoptan por consenso o votación. }\end{array}$ \\
\hline & & $\begin{array}{l}\text { Los consorcios, donde la pertenencia está asociada a la propiedad. } \\
\text { Tienen por finalidad la administración de espacios comunes } \\
\text { del bien que se administra, el ingreso está restringido a ciertos } \\
\text { requisitos, pero las decisiones son consensuadas y la dirección se } \\
\text { elige por votación. }\end{array}$ \\
\hline
\end{tabular}

Fuente: Krieger (2001).

Las organizaciones son básicamente organizaciones sociales que se diferencian entre sí por los fines que persiguen, los roles que ocupan las personas en ellas, las funciones que desempeñan y las interacciones que allí tienen lugar y que conforman una cultura organizacional, donde coexisten normas, costumbres, valores, creencias, principios que marcan un estilo, una identidad, un modo de ser que le es propio, único e irrepetible.

Es en ese espacio físico o virtual donde los sujetos interactúan entre sí y se relacionan con procesos de diverso tipo para contribuir al logro de los objetivos de ese ente organizativo. Allí se presentan situaciones de administración de recursos, implementación de tecnologías, prácticas de persuasión, influencia, obediencia, liderazgo, trabajo en equipo, 
socialización, orden y disciplina, de información y fundamentalmente todas ellas se presentan en acciones de comunicación.

\section{La especificidad de la comunicación en las organizaciones}

Llegado a este punto, es posible afirmar que independientemente de los contextos organizacionales propios de cada una de esas tipologías antes expuestas, la comunicación adquiere una presencia insoslayable, imprescindible y protagónica de la comunicación. Sistematizada, planificada, organizada de manera consciente o asistemática y contingente, constituye un recurso cotidiano irreemplazable al momento de entrelazar procesos internos y externos que faciliten cualquier gestión organizacional.

Sostener que la labor de un comunicador social al interior de la misma es asumir su intervención como un factor sustancial en el monitoreo y gestión de la información y la comunicación (en tanto insumos fundamentales de la administración de las organizaciones) es, desde la fundamentación que aportan las matrices teóricas del funcionalismo y el empirismo analítico, una cuestión cuanto menos necesaria y conveniente.

Su actuación no debiera limitarse a lo interno, sino también a lo externo, por cuanto su accionar facilita la coordinación y la articulación de los recursos para alcanzar objetivos disminuyendo imprevistos y controlando la complejidad, fundamentalmente en un contexto como el actual, donde las organizaciones, tanto como los individuos, se encuentran sujetas a numerosas presiones, cambios y transformaciones socio culturales, tecnológicas, financieras, económicas originadas en el entorno donde se desenvuelven pero que impactan en la dinámica interna, situación que exige una adecuación permanente que les permita mantenerse vigentes en la actividad.

La comunicación en las organizaciones puede ser abordada desde un enfoque profesionalista que concibe a esta rama "como una actividad profesional (la gestión de las actividades a desarrollar y los medios para hacerlo), o desde un enfoque más académico, proveniente principalmente del ámbito universitario, incide en la idea de disciplina científica (la comunicación como una función transversal y fronteriza en las organizaciones)" (Capriotti, 2009: 38).

De manera análoga, es posible encontrar diferentes denominaciones: "comunicación global" (Weil, 1992; Calvo y Reinares, 2001), "comunicación corporativa" (Costa, 1995, 2006); "comunicación integrada" (Capriotti, 1999), "corporativa" (Villafañe, 2002) o "comunicación organizacional” (Prieto Castillo, 1996, 2005), “comunicación institucional” (Kaplún, 2003). Todas ellas tienen en común una mirada holística e integrada de la comunicación organizacional y ponen en valor la importancia de planificar todas las acciones y articular todos los recursos comunicacionales para generar un criterio de trabajo sistémico, que permita unificar misión, visión y objetivos organizacionales y/o institucionales en un marco de eficiencia y eficacia frente a los vínculos internos y externos. Por esto, y a los fines de esta investigación, se adhiere a la conveniencia de crear un sistema global de comunicación entre una organización y sus públicos. 
En términos de Capriotti (2009: 39),

"un sistema global de comunicación no se refiere a una técnica o conjunto de técnicas de comunicación concretas, sino a una estructura compleja, específica y particular de relaciones que tiene una organización con sus diferentes públicos, en la que se utiliza un conjunto abierto de acciones para generar un flujo de información en las dos direcciones entre los sujetos de la relación (organización-públicos), que permitan alcanzar los objetivos establecidos por ambas partes. Así, queda de manifiesto que la comunicación de una organización no tiene un enfoque puramente persuasivo (como instrumento de la organización para orientar la opinión de los públicos), sino fundamentalmente una perspectiva más orientada hacia un enfoque relacional (la comunicación como una forma de "poner en contacto" a la organización y sus públicos)".

Este posicionamiento teórico permite argumentar acerca de la conveniencia para el sistema organizacional de contar con la participación de un comunicador social como bisagra o nexo articulador de las dinámicas internas-externas que son portadoras de sentido para llevar a cabo exitosamente la actividad, ya se trate de cuestiones vinculadas con la gestión al interior como al exterior de la organización.

Es un aspecto que reviste particular interés para esta investigación por cuanto desde lo institucional-académico la comisión curricular de la carrera en cuestión ha planificado la formación de estos profesionales pensando en una matriz conceptual y práctica que habilita a estos profesionales a desempeñar un rol altamente participativo, dinámico, flexible y adaptativo a las circunstancias y a contextos cambiantes como los actuales.

Un criterio que se corresponde plenamente con un modo de concebir el trabajo integrado del comunicador social en los diversos procesos organizacionales en los que su "saber-hacer" complementen la labor interdisciplinaria que estos requieran, pero también aportando los conocimientos específicos que orienten las acciones hacia una mejor interactuación de la organización con los diversos destinatarios de sus acciones y de sus mensajes.

En su formación curricular se previó que contaran con el bagaje teórico y las herramientas metodológicas necesarias para lograr una integración exitosa en los equipos de trabajo. Las destrezas y capacidades adquiridas lo habilitan para insertarse en organizaciones y abordar profesionalmente la comunicación, adecuando las prácticas a la especificidad de cada sistema social, asumiendo la complejidad y la multi-dimensionalidad de la comunicación.

Massoni (2011) postula una concepción innovadora de pensar e intervenir en comunicación, a través de la comunicación estratégica. Concibe al comunicador social

"como un profesional con capacidad de interpelar la dinámica social, para operar crítica y valorativamente en su dimensión comunicativa [...] la especificidad del comunicador es generar espacios de encuentro como una búsqueda que se propone articular prácticas de comunicación y movimientos sociales [...] El comunicador es un profesional capaz de diseñar una acción 
de comunicación integrando todo el proceso, desde la idea a la realización, en función de un objetivo" (Massoni, 2011: 62).

Esta perspectiva amplía aún más el campo de inserción de estos profesionales y la comunicación en las organizaciones adquiere así una posición innovadora, expansiva y de gran vigencia y actualidad. Implica ampliar la limitada asociación que los relaciona sólo con la labor en los medios, como ejecutores de prácticas de comunicación mediática: la visibilidad de las actividades de una organización según su aparición en los diferentes medios masivos de comunicación, a través de la publicidad, la publicación de los partes de prensa, las publi-notas, entre otras, porque gran parte de los procesos comunicacionales son interpersonales, grupales e inter organizacionales y existen numerosos recursos tanto o más ricos, eficientes e idóneos que los mediados.

En este sentido, es pertinente obtener datos que permitan advertir hasta que punto en las organizacionales locales se conoce el alcance de esta disciplina, si incorporaron a esos profesionales para ampliar esos horizontes de vinculación inter e intra organizacional a partir de la implementación de acciones concretas de comunicación.

\section{Análisis de datos}

\subsection{Características organizacionales generales}

El 50\% de las organizaciones bajo estudio fueron casas centrales, el 29\% sucursales, el 4\% delegaciones y un $18 \%$ están incluidas en otro tipo de organización. La actividad principal se muestra en el siguiente gráfico:

Figura 2: Actividad principal.

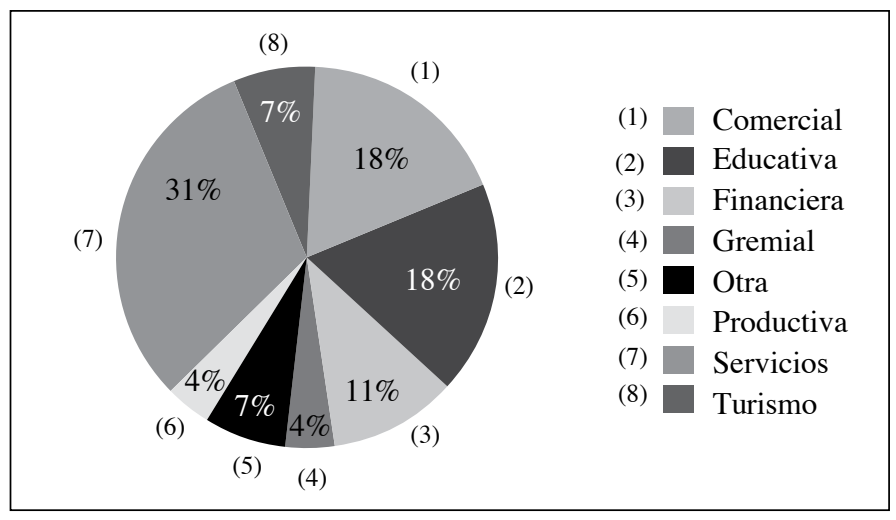

Fuente: Elaboración propia.

Para la variable "tipo de organización" se consideraron las categorías: organizaciones privadas, estatales y mixtas. La muestra analizada reflejó que un 64\% son privadas, 
$25 \%$ estatales y $11 \%$ mixtas. La variable "estructura organizacional" (el modo en que esos organismos establecieron su administración interna) muestra que un alto porcentaje (64\%) está organizada en áreas o sectores, $25 \%$ en otras y entre $7 \%$ y $4 \%$ en gerencias o departamentos. La delegación de actividades de comunicación indica una gran variedad de denominaciones y relaciones funcionales. Esto permite inferir la ubicación estructural y la correlación en función a la toma de decisiones de quien detenta ese cargo.

Existe diversidad de designaciones, ausencia de denominaciones e incluso desconocimiento sobre el nombre que recibe el área en la que se delega la función de comunicación: "área de prensa y difusión", "secretaría", "línea de trabajo en comunicación”, “coordinación”, "área de relaciones institucionales", "recursos humanos", "gerencia", "comunicación interna", "gerencia administrativa", entre otros.

Para la dimensión "profesionales que desempeñan actividades de comunicación", se consideró la pertinencia teórica que se definió en este trabajo y se listaron profesiones afines a la actividad: "licenciados en ciencias de la comunicación" o "comunicación social"; en "ciencias de la información" o "periodismo"; en "marketing y publicidad", en "relaciones públicas", en "marketing", en "medios audiovisuales", en "recursos humanos", en "administración" y "otras". En el 54\% de las organizaciones, estas actividades no están delegadas en profesionales. En el 36\% de los casos se delega en otros profesionales y sólo un $11 \%$ en un licenciado en comunicación social.

\subsection{Acciones de comunicación}

Esta dimensión se desglosó en recursos de comunicación interna (tradicionales y tecnológicos) y de comunicación externa. Se consultó también sobre los medios de comunicación que utilizan para llevar adelante estas últimas.

\subsection{Comunicación interna}

La totalidad de las organizaciones consultadas emplean recursos de comunicación interna (figura 3) en los siguientes porcentajes: 
Figura 3: Comunicación Interna. Empleo de recursos tradicionales.

\begin{tabular}{|c|c|c|}
\hline TIPO & CATEGORÍA & $\%$ \\
\hline Cartelera & & $21 \%$ \\
\hline Manual de política de RR. HH. & \multirow{14}{*}{ Sí emplea } & $21 \%$ \\
\hline Manual de procedimientos & & $30 \%$ \\
\hline Reuniones & & $79 \%$ \\
\hline Desayunos de trabajo & & $42 \%$ \\
\hline Encuestas de clima interno & & $12 \%$ \\
\hline Balance Social & & $91 \%$ \\
\hline Buzón de comunicaciones & & $12 \%$ \\
\hline Memos y circulares & & $36 \%$ \\
\hline Folletos & & $36 \%$ \\
\hline Cartas de bienvenida & & $3 \%$ \\
\hline Capacitaciones & & $58 \%$ \\
\hline Seminarios & & $9 \%$ \\
\hline Talleres & & $27 \%$ \\
\hline Gacetillas & & $24 \%$ \\
\hline
\end{tabular}

Fuente: Elaboración propia.

El 79\% de las organizaciones emplea recursos de comunicación interna mediados por tecnologías de la comunicación y el $21 \%$ no. La distribución de empleo de esos recursos de comunicación interna se grafican en la siguiente figura:

Figura 3: Comunicación Interna. Empleo de recursos tecnológicos.

\begin{tabular}{|c|c|c|}
\hline TIPO & CATEGORÍA & $\%$ \\
\hline Intranet & \multirow{9}{*}{ Sí emplea } & $42 \%$ \\
\hline Blogs & & $12 \%$ \\
\hline Email & & $70 \%$ \\
\hline Boletín electrónico & & $21 \%$ \\
\hline Foros & & $12 \%$ \\
\hline Buzón electrónico de sugerencias & & $15 \%$ \\
\hline Facebook & & $15 \%$ \\
\hline Twitter & & $6 \%$ \\
\hline Skype & & $6 \%$ \\
\hline
\end{tabular}

Fuente: Elaboración propia. 


\subsection{Comunicación externa}

La consulta sobre acciones de comunicación externa arrojaron los siguientes resultados:

Figuras 3 y 4: Comunicación externa. Marketing y merchandising.
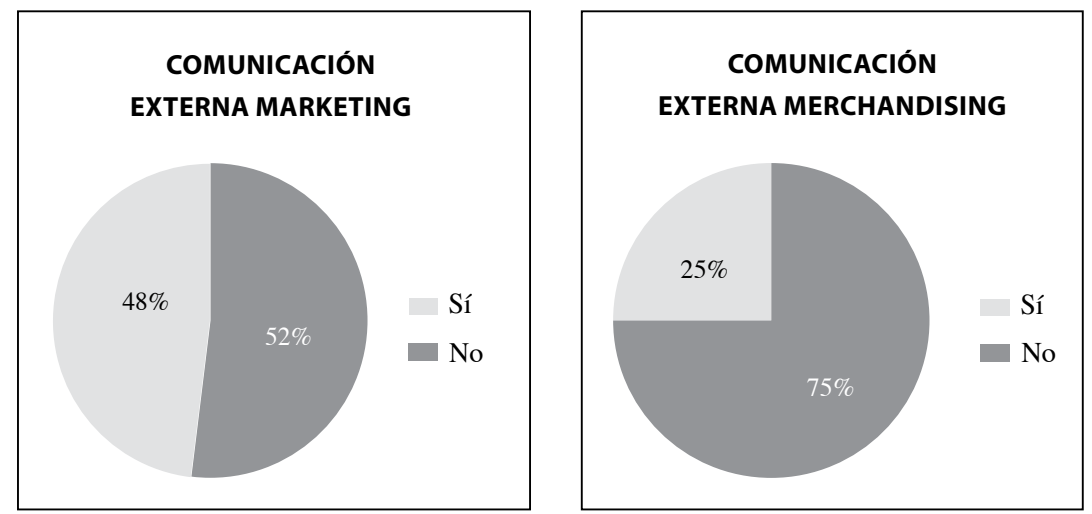

Fuente: Elaboración propia.

"Relaciones con la prensa" (figura 6) y "página web" (figura 7) fueron las actividades de comunicación externa más frecuentes entre las organizaciones. En tanto, los datos relevados de la variable "protocolo y ceremonial" (figura 9) reflejan que un $21 \%$ de las organizaciones realizan acciones relacionadas con ellas.

Figuras 5 y 6: Comunicación financiera y relaciones con la prensa.
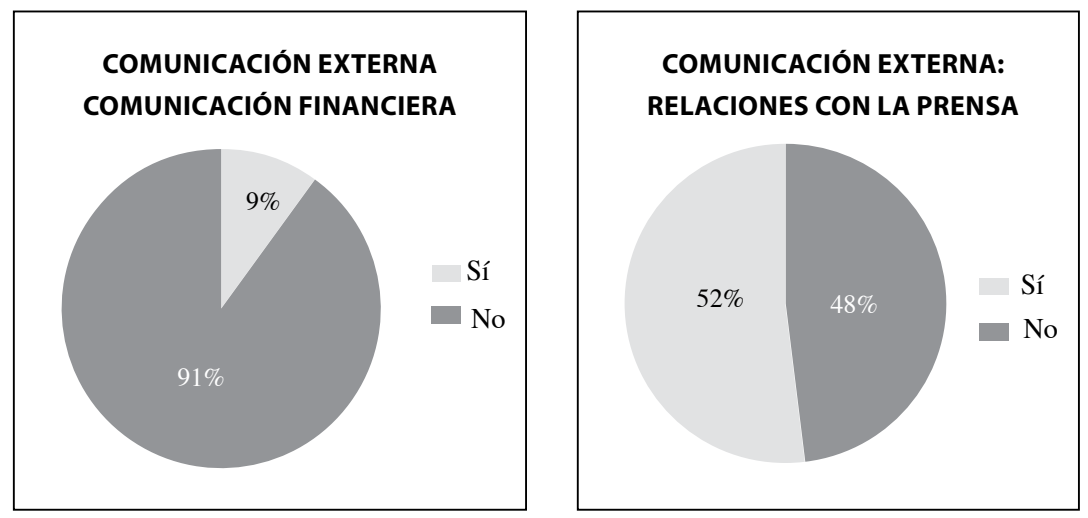

Fuente: Elaboración propia.

En el caso de "mecenazgo" (figura 8), se muestra un porcentaje muy bajo de aplicación, ya que sólo se concreta en un $12 \%$ (sólo cinco organizaciones). Esto puede estar relacionado con el escaso conocimiento o interés que esta práctica comunicacional despierta en aquellas. 
Figuras 7 y 8: Comunicación externa. Página web y mecenazgo.
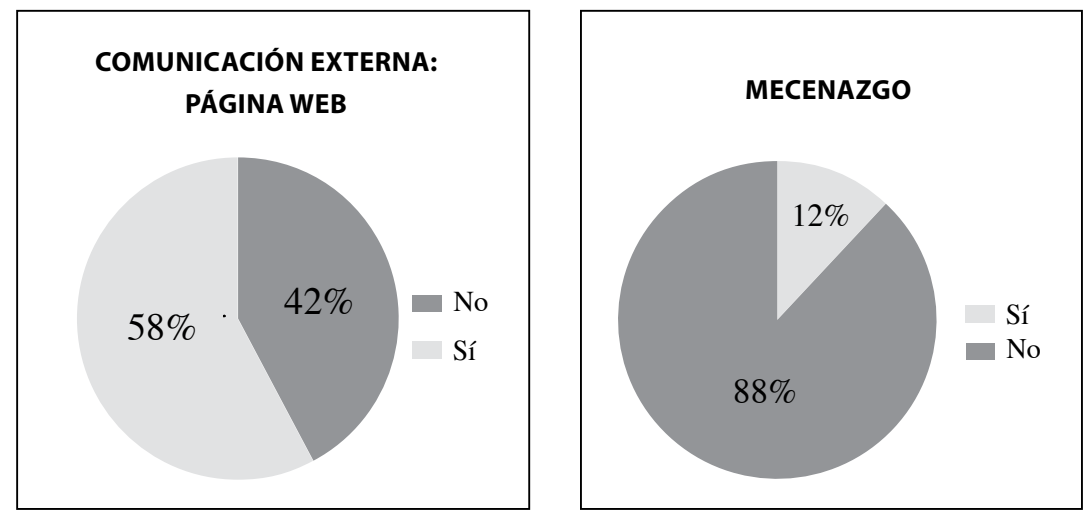

Fuente: Elaboración propia.

Finalmente, Relaciones Públicas (figura 10) es una acción que aplica en un 33\% de la población seleccionada de organizaciones.

Figuras 9 y 10: Protocolo y ceremonial y relaciones públicas.
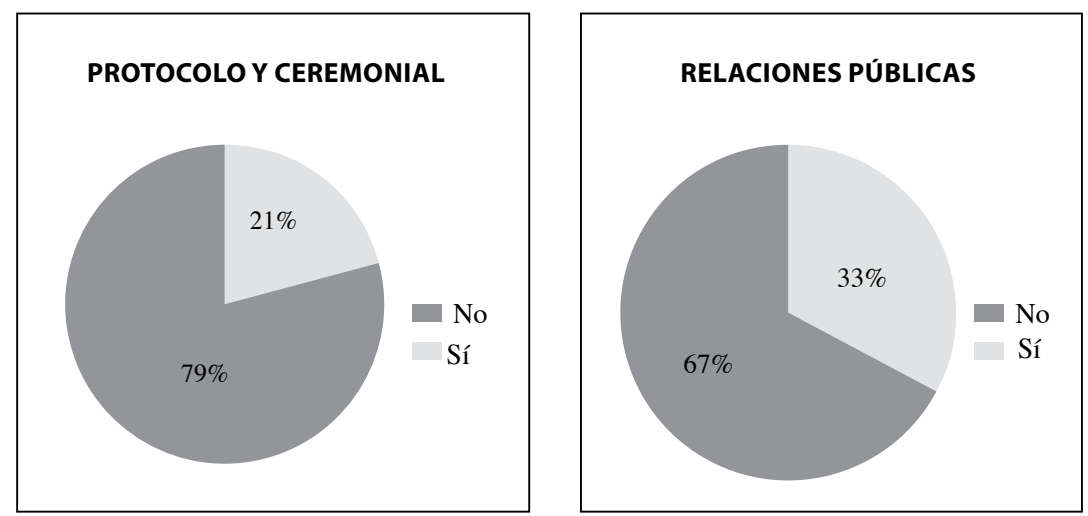

Fuente: Elaboración propia.

\subsection{Medios de comunicación}

En esta categoría se seleccionaron las variables referidos a los medios de comunicación masivos: "radiofónicos", "televisivos", "gráficos" y "digitales". El 82\% de las organizaciones emplea alguno de ellos. Los medios radiofónicos son los más usados $(67 \%$ de las organizaciones), seguidos por los medios gráficos (64\%), los digitales (58\%) y por último los televisivos (45\%). 


\subsection{Demanda de licenciados en comunicación social}

Uno de los objetivos de esta investigación fue conocer la demanda organizacional de licenciados en comunicación social (LCS) en la ciudad de Chilecito. Resultan llamativos los datos recabados (figura 11), ya que el 89\% respondió que consideraba que un profesional con esta formación podía contribuir a mejorar la gestión, un $11 \%$ no sabía o no contestó y ninguno dijo no necesitarlo.

Figura 11: Demanda potencial de LCS.

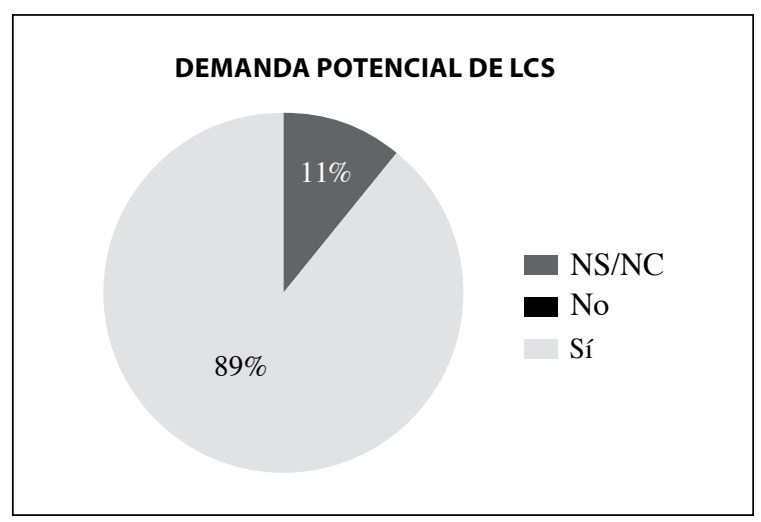

Fuente: Elaboración propia.

Es significativo el elevado porcentaje de organizaciones que consideraron positivo el ingreso a su staff de estos profesionales.

\section{A modo de conclusión}

La investigación que se llevó adelante permitió vincular “comunicación-educación” desde una perspectiva inter-institucional, por cuanto (por una parte) acercó a la universidad, y específicamente a la licenciatura en comunicación social, con el conjunto de las organizaciones del medio en el que posteriormente se insertan sus egresados. Este aspecto fue de por sí un logro, por cuanto permitió conocer la realidad socio-organizacional e identificar la demanda de profesionales de la disciplina.

Permitió también relevar las necesidades de comunicación interna-externa y los modos de resolverlas que implementan los organismos públicos y privados de la ciudad de Chilecito, un insumo informativo valioso para la gestión académica por cuanto permite planificar acciones que promuevan el conocimiento de la disciplina y la pertinencia del perfil profesional en procesos organizacionales diversos.

Se pudo conocer que se emplean gran parte de los recursos de comunicación tradicionales y tecnológicos más frecuentes. No obstante varios de ellos (encuestas de clima interno, 
buzón de comunicaciones, cartas de bienvenida, seminarios) no se utilizan y constituyen una herramienta que facilita la inserción de los públicos internos en la organización, la interactuación funcional, la transmisión de información y la vinculación interna entre los miembros. Los medios tecnológicos más empleados son el correo electrónico e Intranet, lo cual muestra que no se aprovechan otros recursos que facilitarían una comunicación ubicua, fluida, rápida y de bajo costo para la organización. En relación con la comunicación externa, existe una correspondencia entre las tipologías organizacionales y las acciones que concretan.

Resulta llamativa la posible demanda de licenciados en comunicación social. Si se tiene en cuenta que las respuestas se nuclearon entre la necesidad de contar en sus organizaciones con un profesional o no saber/no contestar en relación con los aportes que estos podrían hacer a sus organizaciones, es posible entonces indicar que 29 organizaciones de Chilecito $(89 \%$ de las 33 consultadas) asumen como necesaria la inclusión de este profesional en su staff.

Una cifra para nada despreciable cuando de promover la inserción de los egresados en el medio se trata. En este punto aparece nuevamente la vinculación "comunicacióneducación" desde una doble perspectiva: la propia de la díada que hace referencia a la formación, las prácticas, los procesos, las metodologías, pero también la necesidad de ampliar la perspectiva hacia la relación fluida que debe mantener la institución educativa con el medio socio cultural en el que se evidencia su razón de ser.

Finalmente, se pudo advertir que el nivel de conocimiento sobre los alcances de la formación de un licenciado en comunicación social y su contribución a la gestión integral del organismo es escaso, contingente, dispar. Pudo inferirse que en las políticas de gestión de la comunicación aplicadas por los organismos públicos y privados de Chilecito no intervienen (y si lo hacen es de modo esporádico) los profesionales con formación específica. Lo medios de comunicación preferidos son los radiofónicos, seguidos de los televisivos.

Estos resultados muestran un estado de situación actual del contexto organizacional en el que se inserta el egresado de la licenciatura en comunicación social de la Universidad Nacional de Chilecito, sobre el cual es posible llevar adelante acciones de difusión de los alcances de la formación de esos profesionales. Permite reconocer que la demanda organizacional aún no está cubierta (en gran medida por desconocimiento) y que las expectativas de abordaje de políticas de inserción laboral son altas.

\section{Fuentes consultadas}

ALAIC (2004). "Papers Grupo de Trabajo de Comunicación Organizacional y Relaciones Públicas". VII Congreso ALAIC. La Plata, Argentina. Extraída el 14/XI/2016 desde www. eca.usp.br/alaic

Álvarez, H. (2003). Administración, un enfoque interdisciplinario y competitivo. Córdoba: Eudecor. 
Amado, A. (1999). Comunicaciones Públicas. El modelo de la comunicación integrada. Buenos Aires: Temas.

Ávila, R. (2004). Crítica de la comunicación organizacional. México, D. F.: UNAM.

Baccin, C. (2003). "Comunicación institucional en Argentina: organizaciones gravitantes y opciones para el campo de conocimiento”. Boletín Temático ALAIC, núm. 11. Extraída el 26/X/2016 desde www.eca.usp.br/alaic

Calvo, S. y Reinares, P. (2001). Comunicación en Internet. Estrategias de marketing y comunicación interactivas. Madrid: Paraninfo.

\section{Capriotti, $\mathbf{P}$.}

_(2009). Branding corporativo. Fundamentos para la gestión estratégica de la identidad corporativa. Santiago de Chile: Libros de la Empresa.

_(1999). Planificación estratégica de la imagen corporativa. Barcelona: Ariel.

\section{Costa, J.}

_(2006). Imagen corporativa en el siglo XXI. Buenos Aires: La Crujía.

(1995). Comunicación corporativa y revolución de los servicios. Madrid: Ciencias Sociales.

Del Pilar, T. (1993). “Desde donde vamos a entender la comunicación organizacional”, pp. 23-28. II Simposio Latinoamericano de Comunicación Organizacional. Cali, Colombia.

\section{Kaplún, G.}

_(2003). "Comunicación organizacional: la importancia de los bordes y las ventajas de agacharse". Extraída el 23/X/2017 desde

https://ccyo.files.wordpress.com/2009/08/comunicacion-organizacional-la-importanciade-los-bordes-y-las-ventajas-de-agacharse-gabriel-kaplun.pdf

_(1999). "Las metáforas de la organización”. Relaciones, núm. 187. Extraída el 23/X/2917 desde http://www.chasque.net/frontpage/relacion/9912/index.html

Krieger, M. (2001). Sociología de las organizaciones. Una introducción al comportamiento organizacional. Buenos Aires: Prentice Hall.

Larrea, J. (2008). Apuntes del DIRCOM. Buenos Aires: DIRCOM.

Manucci, M. (2004). "La incertidumbre como espacio de intervención en Comunicación Corporativa". Extraída el 23/X/2017 desde http://www.razonypalabra.org.mx/libros/libros/incertidumbre.pdf

Massoni, S. (2011). Comunicación estratégica, comunicación para la innovación. Rosario: Homo Sapiens. 
Mayntz, R. (1980). Sociología de la organización. Madrid: Alianza.

Moragas, M. (2011). Interpretar la comunicación. Estudios sobre medios en América y Europa. Barcelona: Gedisa.

Muriel, M. y Rota, G. (1980). Comunicación institucional. Enfoque social de las relaciones públicas. Quito: CIESPAL.

Peiró, J. y Bresó, I. (2012). “La comunicación en las organizaciones: una aproximación desde el modelo de análisis multifacético para la gestión y la intervención organizacional (modelo Amigo)". Persona, núm. 15, pp. 41-70. Extraída el 23/X/2017 desde http://fresno. ulima.edu.pe/sf\%5Csf_bdfde.nsf/imagenes/3AFA3911E143A81F05257AFA0060089B/\$f ile/02-persona15-peiro.pdf

Pineda, M. (2001). "Qué investigar hoy sobre Comunicación en América Latina?". Diálogos de la Comunicación, núm. 62, pp. 77-83. Extraída el 23/X/2017 desde http://www.dialogosfelafacs.net/wp-content/uploads/2012/01/62-revista-dialogos-queinvestigar-hoy-sobre-comunicaci $\% \mathrm{C} 3 \% \mathrm{~B} 3 n$-en-america-latina $\% \mathrm{EF} \% 80 \% \mathrm{~A} 5$.pdf

Prieto Castillo, D. (1993). Planificación de la comunicación institucional. San Salvador: Universidad Centroamericana José Simeón Cañas.

Rodríguez, D. (2006). Diagnóstico organizacional. México D. F.: Alfaomega.

Schein, E. (1988). La cultura empresarial y el liderazgo. Una visión dinámica. Barcelona: Plaza \& Janés.

Schvarstein, L. (1998). Diseño de organizaciones. Tensiones y paradojas. Buenos Aires: Paidós.

Villafañe, J. (2002). Imagen positiva. Gestión estratégica de la imagen de las empresas. Madrid: Pirámide.

Weil, P. (1992). La comunicación global. Barcelona: Paidós. 